\title{
OPTOMECHANICS
}

\section{Light-driven flytrap}

The realization of microrobotic devices that mimic living creatures is a growing trend and a popular topic of research. To date, most demonstrations have relied on computercontrolled electric actuation of moving parts. However, the complex circuitry and power sources involved often make devices difficult to fabricate. Now, inspired by the well-known insect-eating plant the Venus flytrap, Owies Wani and co-workers from Tampere University of Technology in Finland have realized a light-driven gripping microdevice (pictured; left) (Nat. Commun. 8, 15546; 2017).

The gripper was made from a thin (20- $\mu \mathrm{m}$-thick) layer of a light-sensitive liquid-crystal elastomer (LCE) containing 2 mol\% N-ethyl-N-(2-hydroxyethyl)-4-(4nitrophenylazo)aniline. The light-induced actuation works by controlling the molecular alignment within the liquidcrystal polymer network. A hole was cut from the centre of the LCE layer and the tip of a multimode optical fibre fixed in place. Blue light from a 488-nm laser was coupled to the other end of the fibre and sent through the centre of the device. The divergence angle of the emitted light from the fibre tip was $13^{\circ}$. If an object is present just in front of the gripper, it causes light to be reflected or scattered back to the LCE layer that absorbs it, causing the layer to mechanically bend towards the object, eventually capturing it (pictured; right).

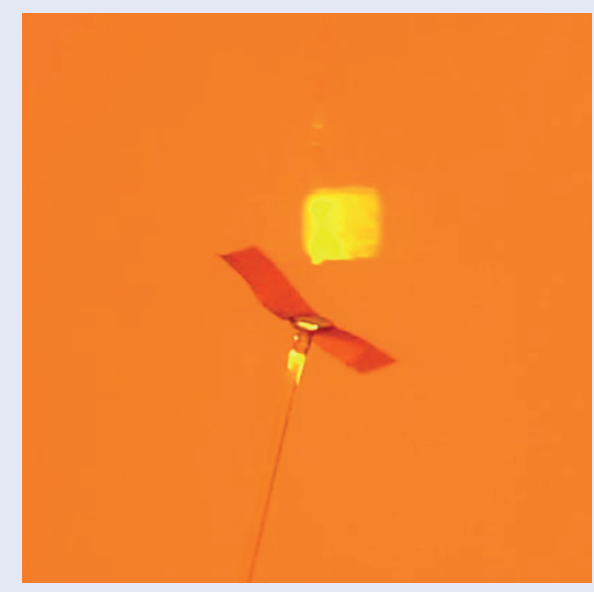

The size of the gripping force is proportional to the excitation light intensity, and for a light level of $100 \mathrm{~mW} \mathrm{~cm}^{-2}$, the force was $250 \mu \mathrm{N}$, which is sufficient to sustain an object with a weight of tens of milligrams. The gripper rapidly releases the object once the light is turned off. The response time of the light-driven flytrap also depends on the light power, with the gripper closing within $200 \mathrm{~ms}$ for light power of about $300 \mathrm{~mW}$.

As for future applications of the optical gripper, one possibility is in production-line monitoring. "The device may potentially be used in manufacturing units that require automatic detection of product defects," explained Wani.

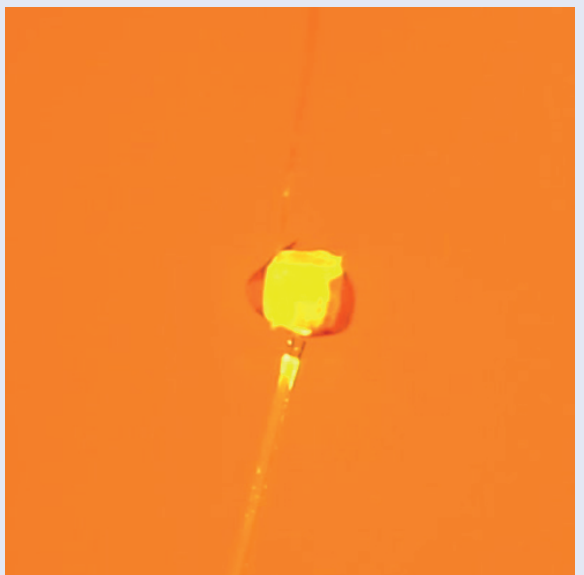

To demonstrate this potential, a set of cube-shaped particles were placed on a translation stage (to mimic a miniature production line) moving beneath the lightdriven flytrap. Once a product that provided sufficient scattering light approached the flytrap, it was automatically selected.

The light-driven flytrap was successfully operated for a number of grip-andrelease cycles. The use of high-power illumination (for example, $200 \mathrm{~mW}$ ) led to irreversibility in the actuation process, but the original shape and functionality could be recovered by annealing the flytrap at a temperature of $45^{\circ} \mathrm{C}$.

NORIAKI HORIUCHI

\section{ATTOSECOND PHOTONICS}

\section{Imaging ultrafast electron dynamics}

A feasible route towards electron microscopy at the attosecond timescale is now possible thanks to the implementation of an optical gating approach.

\section{Alberto Simoncig}

$\mathrm{P}$ roficiency in designing the next generations of functional materials is strictly dependent on the ability to unlock the behaviour of matter at the nanoscale, as well as to control it at fundamental timescales. To satisfy this ambition, it is mandatory to engineer the quantum properties of matter in a way that they are tangible for technological applications ${ }^{1}$. In this view, future functional materials will offer the unique possibility to design devices that are able to achieve breakthroughs and facilitate a broad range of applications spanning from fundamental science to the technological, industrial and defence sectors, interconnecting several branches of knowledge.
The main stumbling block for current device technologies is their intrinsic architecture based on electron-transport processes, which sets a limit to pushing operating clock frequencies towards faster regimes $^{2}$. Addressing this issue will be dependent on the capability to take realtime snapshots of the behaviour of matter at timescales where fundamental processes 